DOI: https://doi.org/10.35560/jcofarts102/177-194

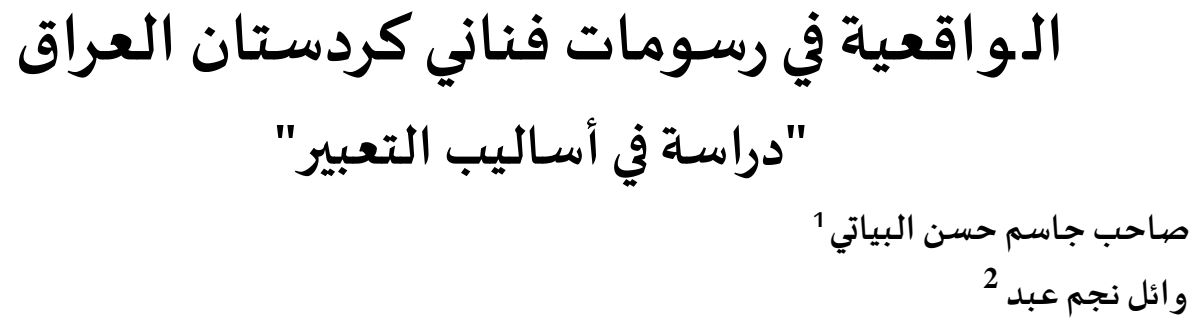

ISSN(Online) 2523-2029, ISSN(Print) 1819-5229 مجلة الأكاديمي-العدد 102-السنة 2021 تاريخ استلام البحث 2021/10/18 تاريخ قبول النشر 2021/11/29 ، تاريخ النشر2021/12/15

تناول البحث الموسوم (الواقعية في رسومات فناني كردستان العراق" دراسة في أساليب التعبير")

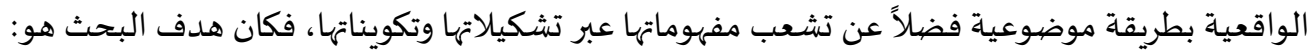
تعرف الواقعية في رسومات كردستان العراق، وقد تضمن البحث أربعة فصول أهتم الفصل الأول بتسليط

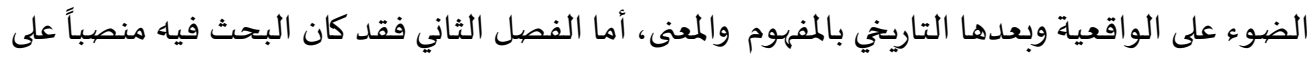
أسـاليب التعبير في الرسم، اما الفصل الثالث فقد عني بـ التطبيقات الأجرائية لأساليب التعبير الواقعية في

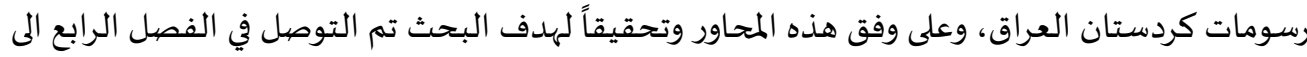
عدد من النتائج نذكر منها: 1-وثقت الواقعية حياة المجتمع الكردستاني بما ينسجم مع تطورات العصر إذ شهد الأسلوب سمة

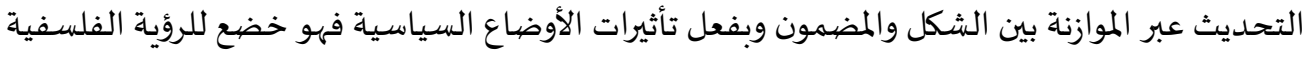
والأفكار الجدلية والمفاهيم. 2-حققت الواقعية في بعدها النقدي اكتشاف للبصيرة الفنية والفنتازيا الحالمة أزاء الأعمال الفنية التي تناولت الرمز والبطولة والأسطورة.

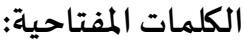
الواقعية، أساليب، التعبير، كردستان العراق، فناني كردستان. تقدمة: لقد أهتمت الواقعية بالدرجة الأسـاس بالواقع المادي المحسوس، ذلك الواقع الذي يتم رصده في المجتمع بطريقة موضوعية، إلا إنها في الواقع تروم الإنفتاح على روح العصر وسيرته التأريخية، إذ لم تعد الواقعية

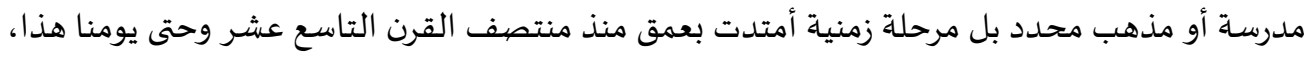




\section{ISSN(Online) 2523-2029, ISSN(Print) 1819-5229 2021 مجلة الأكاديمي-العدد 102-السنة}

إذ لم يُعد الأسلوب الواقعي يهتم بالمحاكاة التسجيلية المباشرة وحسب بل تعدى الأمر إلى تشعب مفهوماتها بواقعيات فنية ابداعية وتأملية- إن جاز التعبير - ولكنها بقيت تمنح الواقع عبر تشكلاتها وتكويناتها بعداً

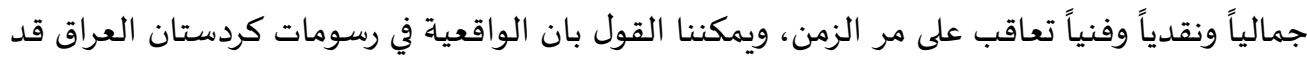

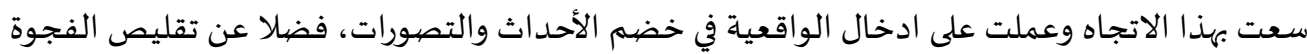
بين ماهو واقعي وخيالي. وعليا يحدد الباحث مشكلة بحثه بالتساؤلات الآتية:

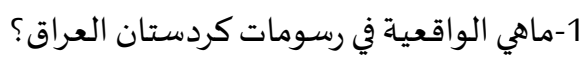

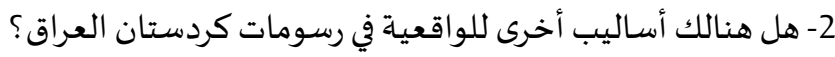
3-هل للمرجعيات البيئية والثقافية والتاريخية لإقليم كردستان دوراً مؤثراً في تحول أساليب التعبير في الواقعية؟

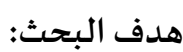
يهدف البحث الحالي إلى: تعرف الواقعية في رسومات كردستان العراق( دراسة في أسـاليب التعبير) مصطلحات البحث:

الواقعية: هي تمثيل الأشياء والعلاقات في الأعمال الفنية، وهي ادراك للحياة الاجتماعية، تثير فينا وعياً

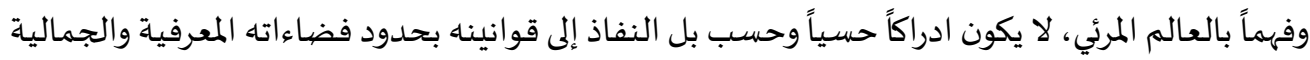
والثقافية والاجتماعية والسحرية.

\section{الفصل الأول: (الـو اقعية المفهوم والمعنى)}

تعد كلمة الواقعية من أكثر المصطلحات تشعباً، وإنها قد اتخذت المعوم وعاني كثيرة ومتنوعة إذ أن الواقعية

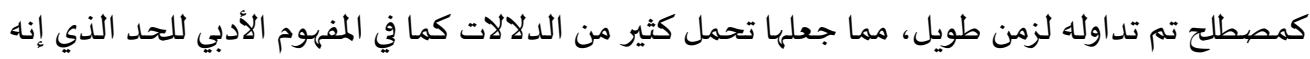

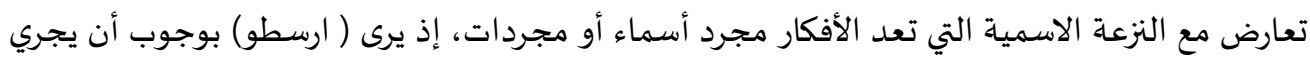

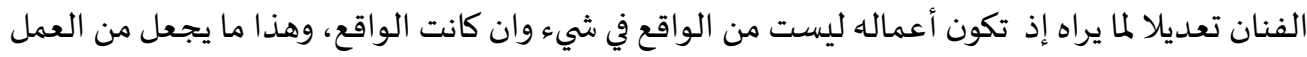

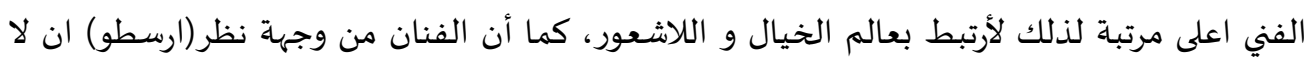

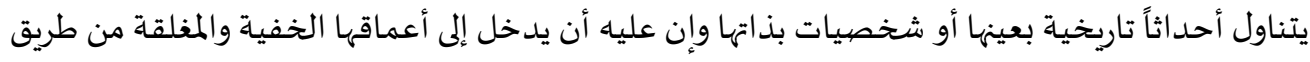

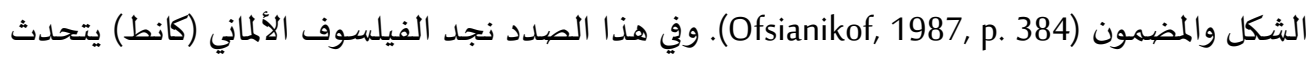
عن (المثالية وواقعية الأهداف الطبيعية) مما جعلنا وجها لوجه أمام الواقعية الفلسفية وهو المثالية التي

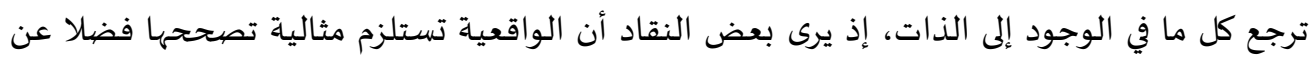

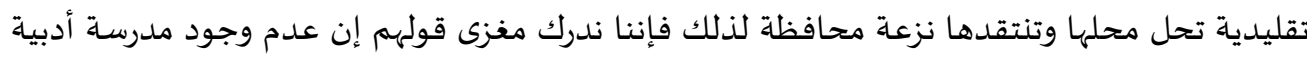

سوى الواقعية، إذ لم يكن أحد على تمام الوعي بعمله مثل الكتاب الواقعيون (Fadl, 1980, p. 20).

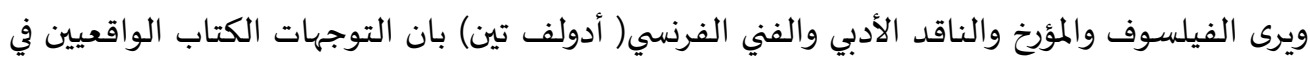

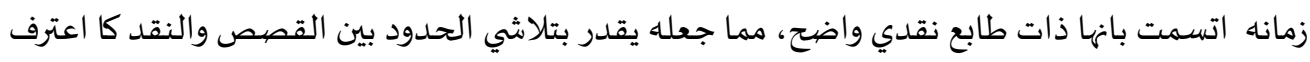

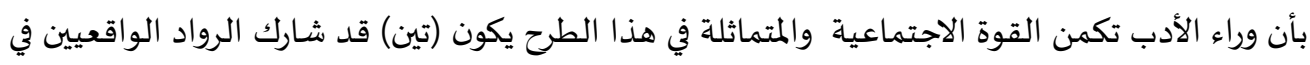

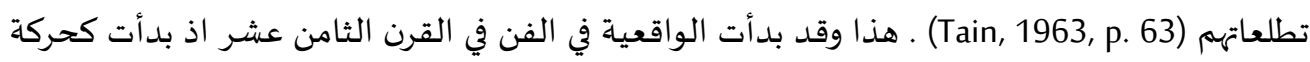
واضحة المعالم والأهداف في الفن و في منتصف القرن التاسع عشر هي الشكل الفني السائد والواقعية وكما 


\section{ISSN(Online) 2523-2029, ISSN(Print) 1819-5229 2021 مجلة الأكاديمي-العدد 102-السينة}

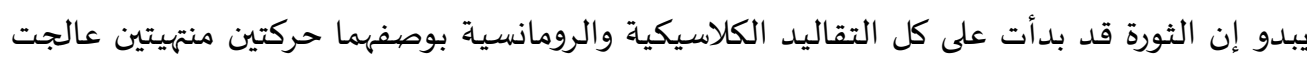

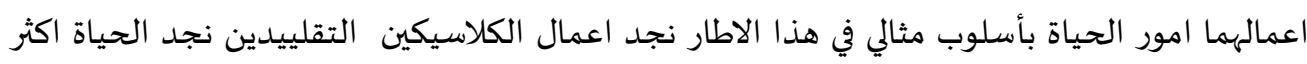

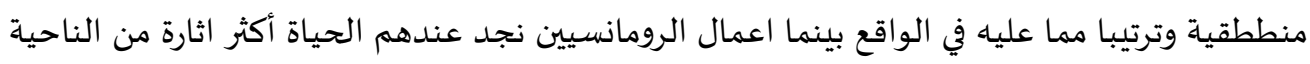

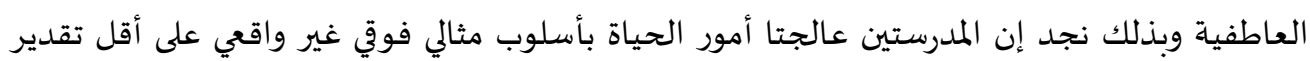

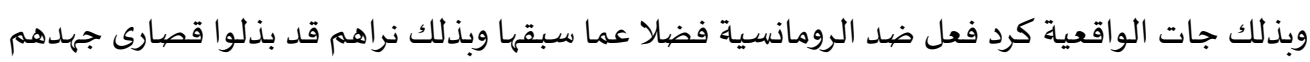

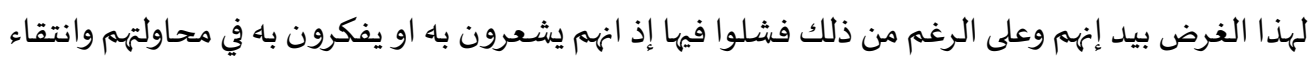

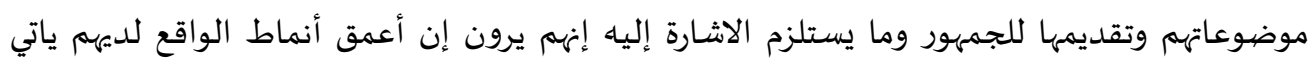

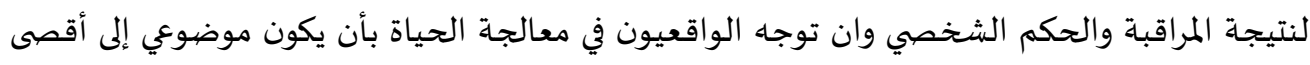
درجة مستطاعة . فقد اشار (روجياء غارودي) في كتابه (واقعية بلا ضفاف) بان الواقعية في الفن هي الوعي بالمشاركة في خلق

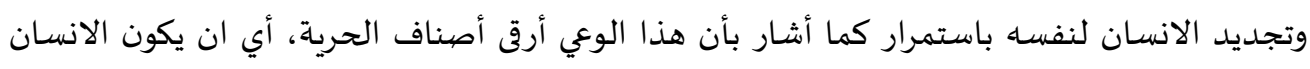

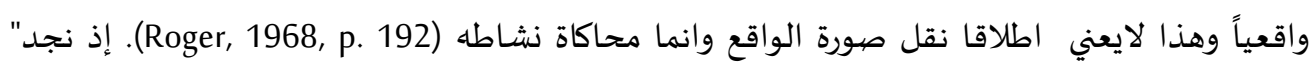

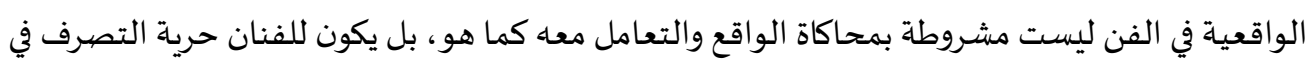

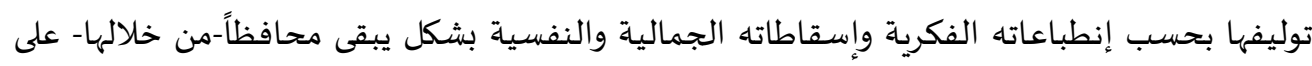
طابعها الواقعي" (Saleh, 2014, p. 46) ويصف (جورج لوكاش) الواقعية فيقول عن الواقع الموضيوعي تدور

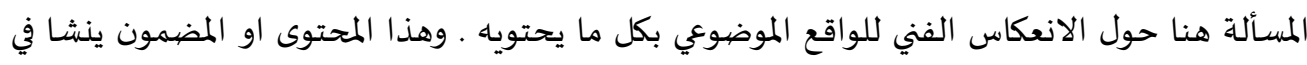

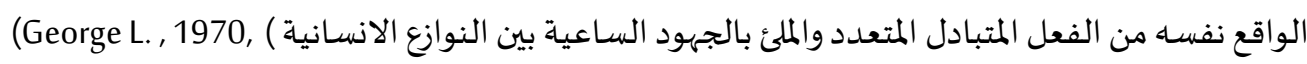

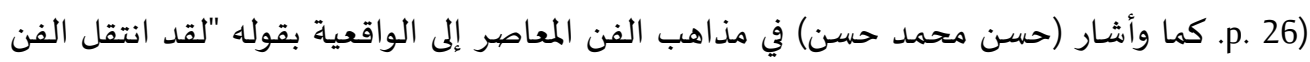

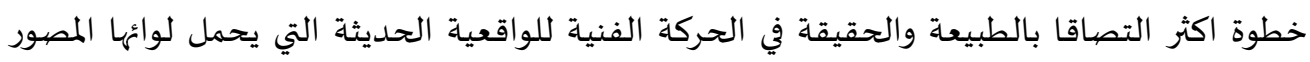

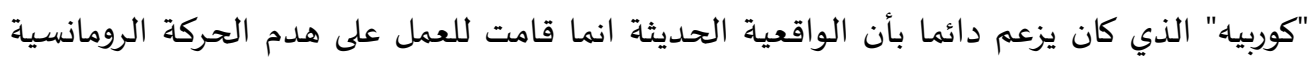
والقضاء عليها إذ إن هذا هو الطريق السليم للوصول إلى الحقيقة والجمال" . (Hassan H. M., p. 16)

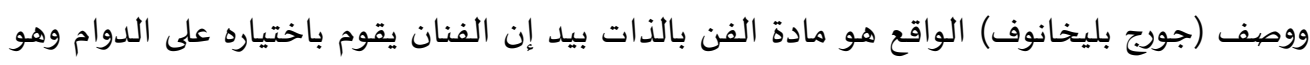

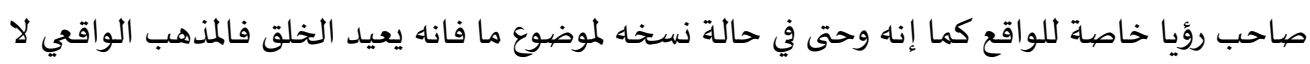

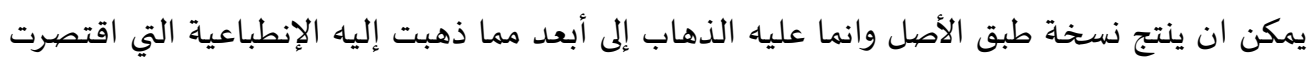

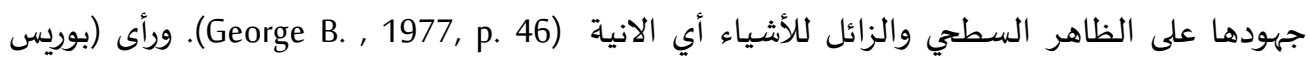

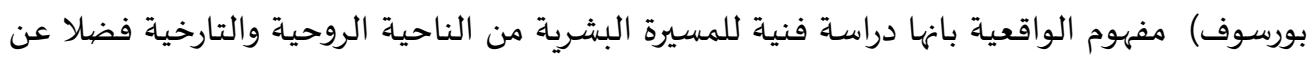

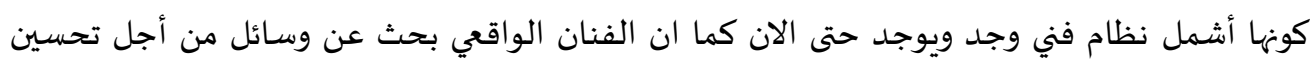

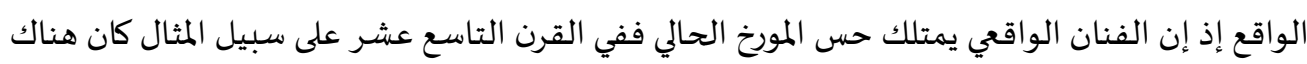

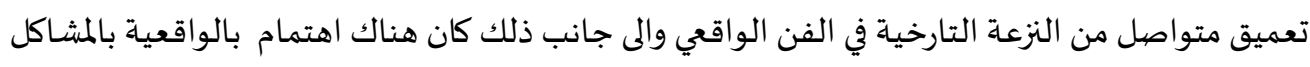
الإنسانية الأكثر شمولية. (Boris, p. 150). فالحركة الواقعية ركزت على العيل المنطق الموضيوعي وجعلته أكثر

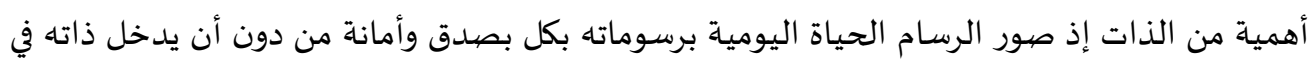

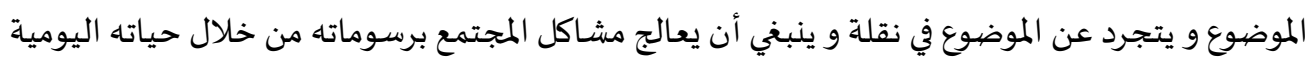




\section{ISSN(Online) 2523-2029, ISSN(Print) 1819-5229 2021 مجلة الأكاديي-العدد 102-السينة}

محاولا إيجاد الحلول لتلك المشكلات ـ ويقول الرسام الأيطالي(كارافاجيو)(1571) أن الواقعية في الفن: هي

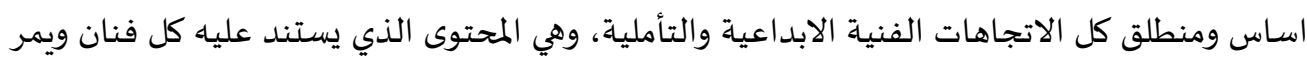
من تحته هي رصيد الفنان وهي مدرسته الأولى، وهي المنبع التي انطلق منها كبار الفنانين لينطلقوا ويأتوا

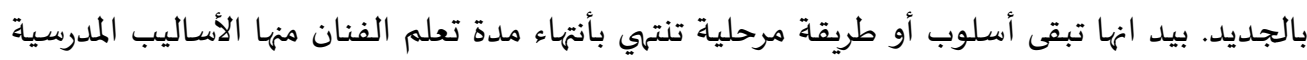

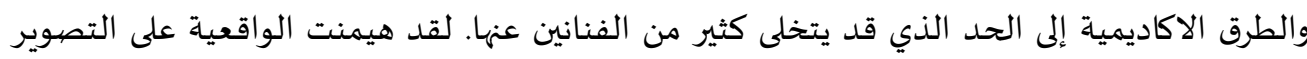
التشكيلي الأوروبي في القرن التاسع عشر، وظهر ذلك جليا في رسومات فنانين فرنسيين، مثل( كاميل كورو)

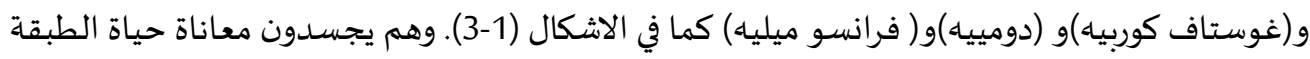

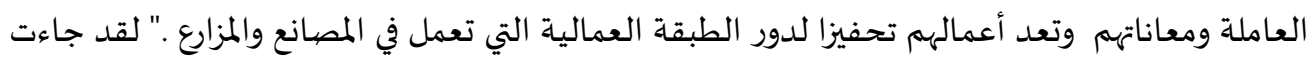

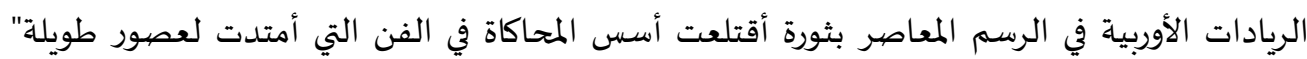

(Mahdi, 2012, p. 67)

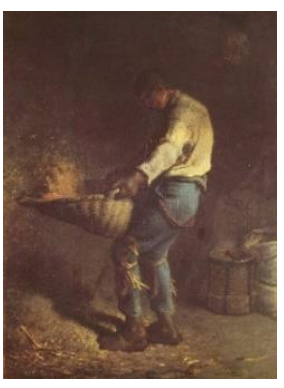

(3) (3) (3)

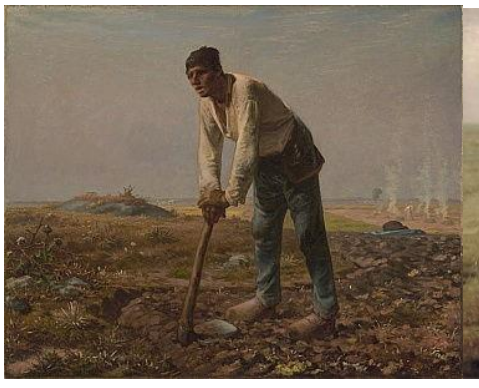

الشكل(2)

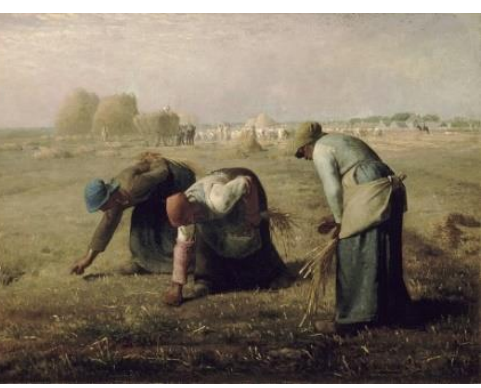

الشكل(1)

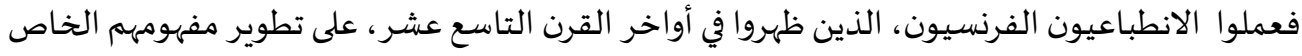

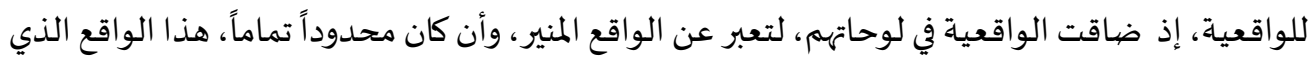

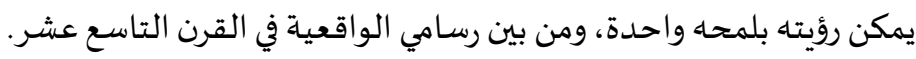
الفصل الثاني: الـواقع واسـاليب التعبير في فن الرسم

ان أسـاليب التعبير في الواقعية لا تقتصر على مرحلة تاريخية محددة لانها ظاهرة تجلت بأشكال متنوعة في

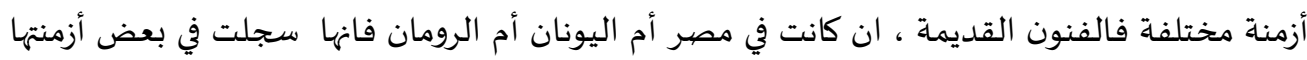

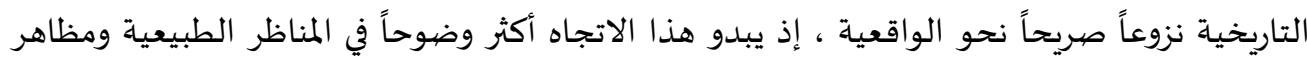

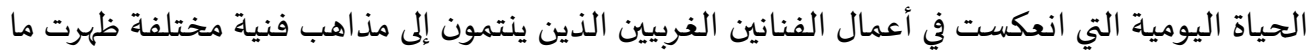
بين القرنين الخامس عشر والتاسع عشر وفي أماكن مختلفة من القارة الأوربية ( النمسا ربلجيكا ،وهيكا ،وهولندا

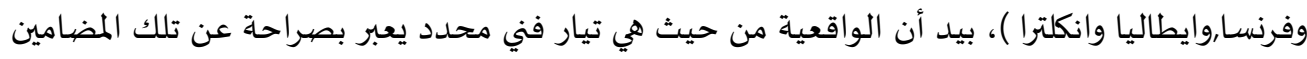

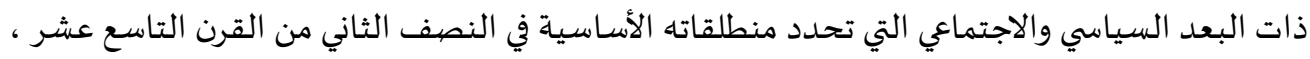

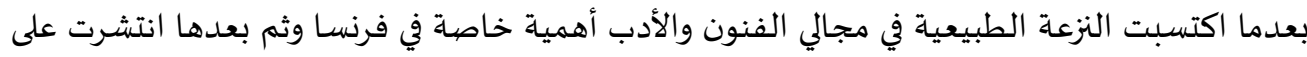

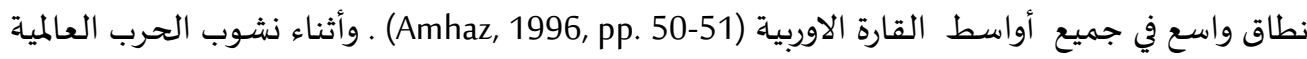
الثانية وفي عام 1942 وظهور بوادر تأثير الفن العراقي بالفن الغربي وتجسدت من خلال مبيء الرسامين 
ISSN(Online) 2523-2029, ISSN(Print) 1819-5229 2021 مجلة الأكاديمي-العدد 102-السينة

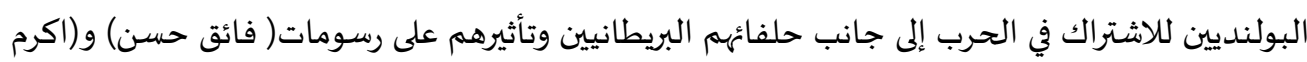
شكري) و(جواد سليم )وغيرهم بالاسلوب ما بعد الانطباعي أو التنقيطي (AL Said, Seasons of plastic) movement in Iraq, 1983, p. 229) وجنوب العراق للكشف عن القيم اللونية وإمكانية التعبير عبر تمثيلات الواقعية التي حاولت أن تتناول

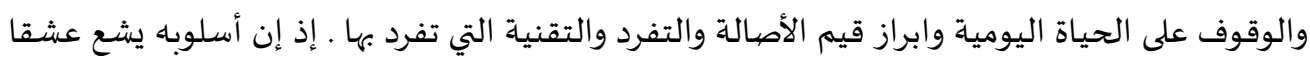

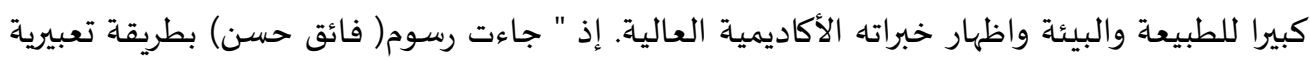

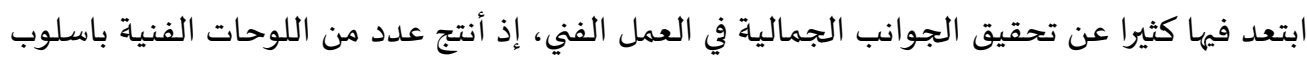

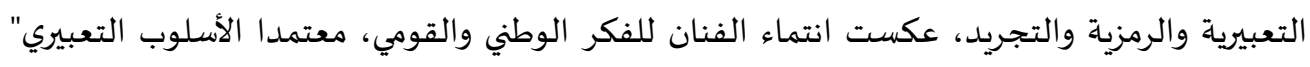
. (Hassan Q., 2016, p. 24) بينما كان طرح( كاظم حيدر) (1932-1985) ذات رؤيا فكرية تراثية تحمل مضامين انسانية درامية والذي تجسـد في معرضها عن ملحمة الشهيد عام (1965) ومفاهيم الاستشهاد لثورة الحسين ( عليه السلام ) كما

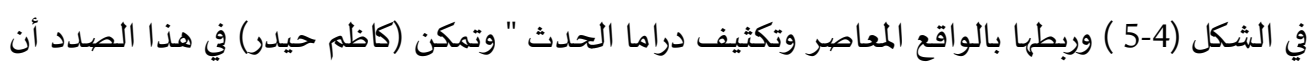

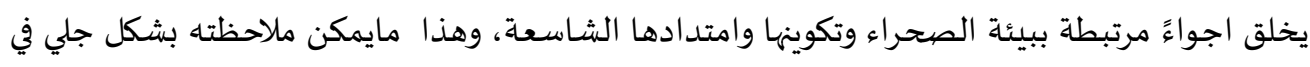

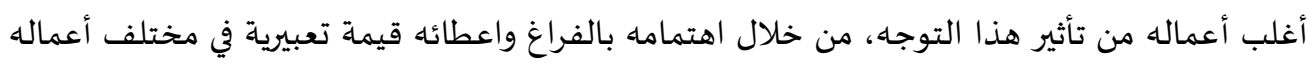
. (Kamel, Iraqi Formation, Foundation and Diversity, 2000, p. 229)

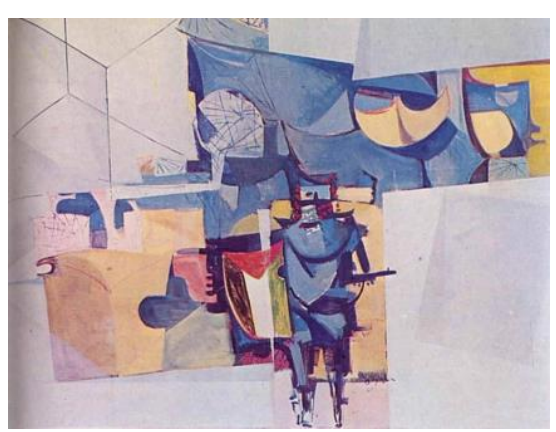

شكل (5)

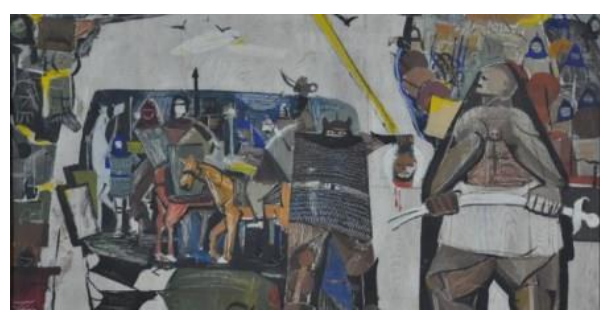

شكل (4)

في حين يُعد اسلوب الفنان( ماهود أحمد )واقعياً تعبيرياً ، إذ يستلهم موضوعاته الفنية ورسوماته التي

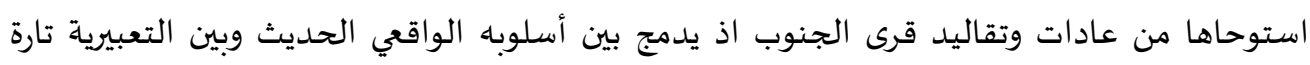
والرمزية تارة أخرى ويزاوج في تحقيقه ضمن اتجاهات وأساليب حديثة ،ولكنها يبحث عن أجواء التي تمكنها

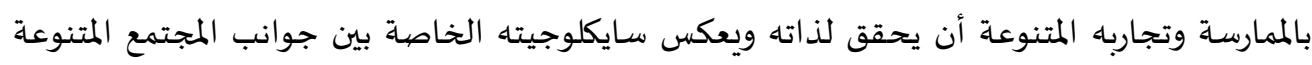

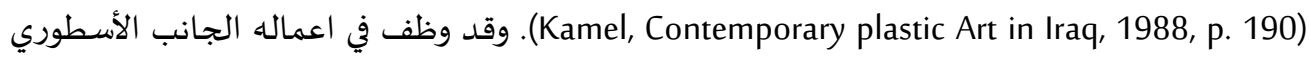
و"اهتمامه الدائم بتسخير الرموز التاريخية في خدمة موضوعه ويوظف أدوات الحس الشعبي لكي يبرز لنا

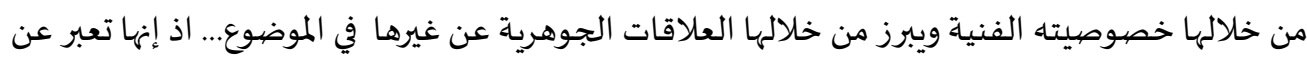
المسائل الإنسانية في كلا الجانبين التاريخي والاجتماعي" (AL-Rubaie, Painting and ideas, 1976, p. 253). 
ISSN(Online) 2523-2029, ISSN(Print) 1819-5229 2021 مجلة الأكاديمي-العدد 102-السينة فأشكال الأسطورة التي تبناها الفنان قد تتخطت علاقاتها الواقعية في النسب والتناسب التي تتلائم مع دور الأحداث كذلك عن تخطي مفهومي الزمان والمكان مما يجعلهما في حاله حركه عبر الزمكان وكذلك الحال

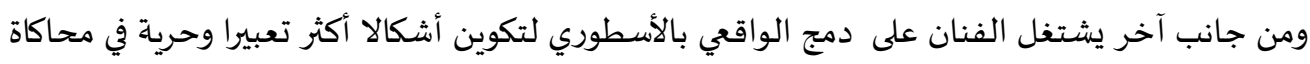

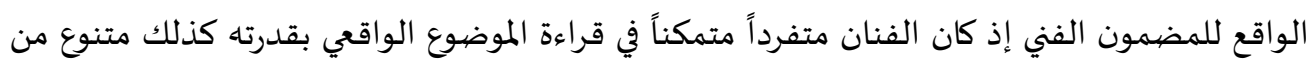

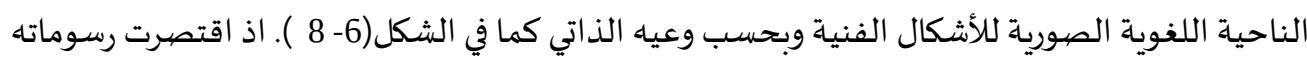

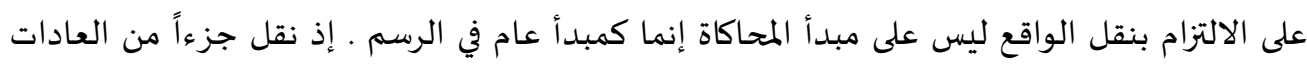

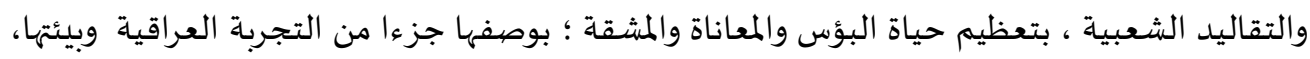

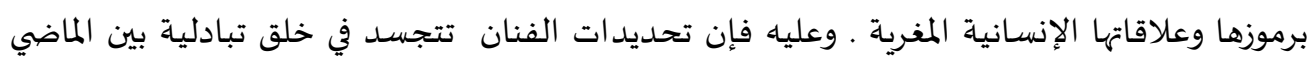

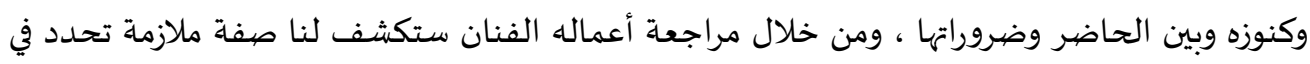

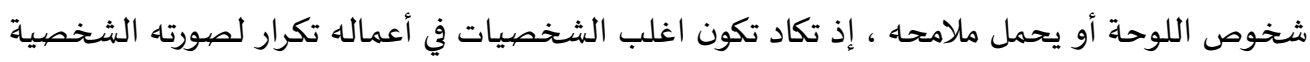

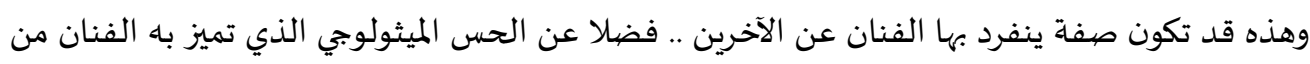
خلال عودته وحنينه للقرية والريف .

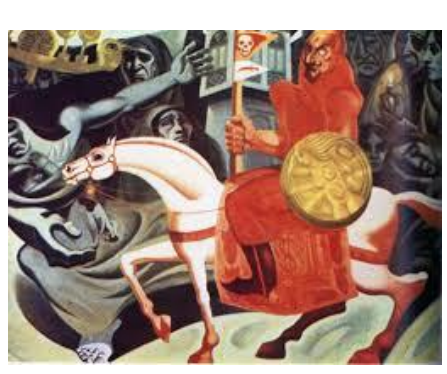

شكل (8)

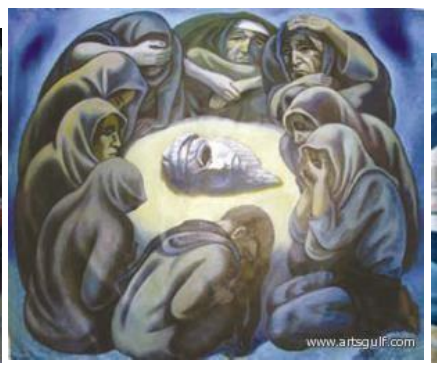

شكل (7)

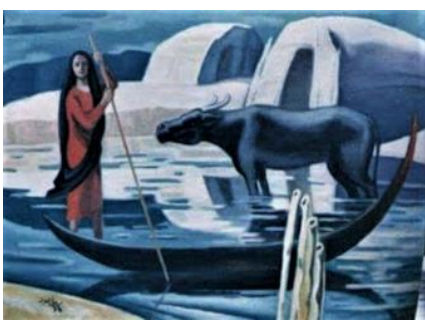

شكل (6)

الفصل الثالث: تطبيقات اجر ائية لإسـاليب التعبير الواقعية في رسومات فناني كردستان العراق: ان الحركة الفنية في كُردستان العراق شأهها شأن الحركات والاتجاهات الفنية بكل المجتمعات والتي

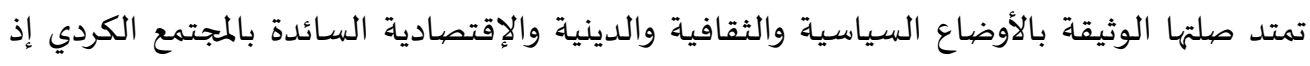

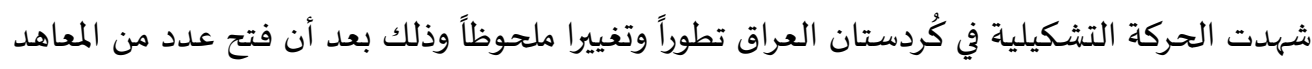

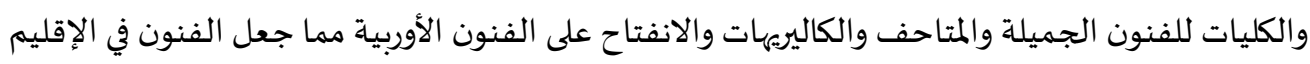

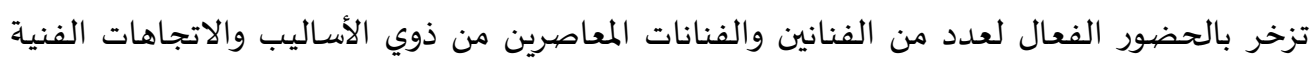

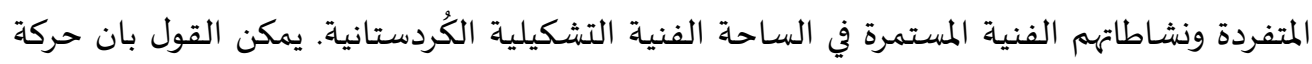
(Yusuf, 2005, p. . الفن التشكيلي المعاصر في كردستان العراق هو امتداد لحركة الفن التشكيلي في العراقيه

1-الـواقعية الطبيعية والبحث عن المحاكاة: إن أول فنان مارس الرسم بشكل واضح كان الفنان (عثمان بك) كان من مدينة السلمانية والذي ظهر ببداية القرن العشرين وكان يمارس هوايته في المقابر أثناء زيارة 
ISSN(Online) 2523-2029, ISSN(Print) 1819-5229 2021 مجلة الأكاديمي-العدد 102-السينة النساء للمقابر اللواتي كان يزورن امواتهن ورسمهن أثناء جلوسهن بين الأعشاب والزهور التي كانت هذه

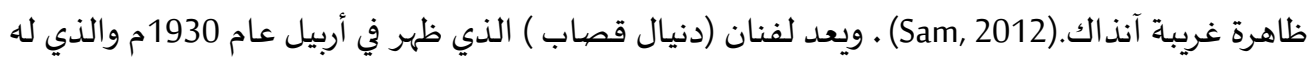

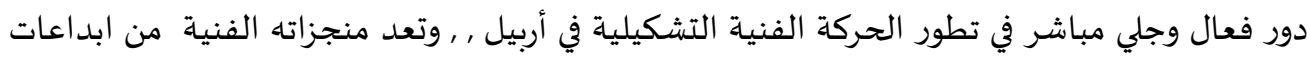

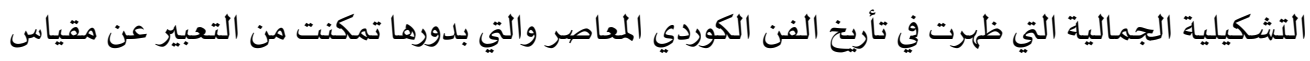
أكاديمية رفيعة جسدت في التعبير عن البيئة الكوردستانية بإبعادها المتعددة (الطبيعية والسياسية

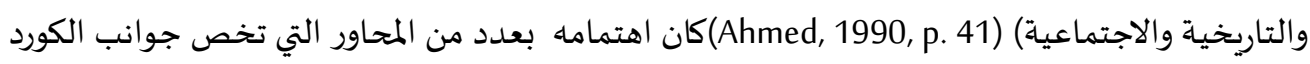
في كوردستان مثل رسم المناظر الطبيعية والمدن والقرى الكوردية وتوثيق المراسيم الاجتماعية مثل:(زيارة النساء للقبور) كما في الاشكال (9-10) مئل

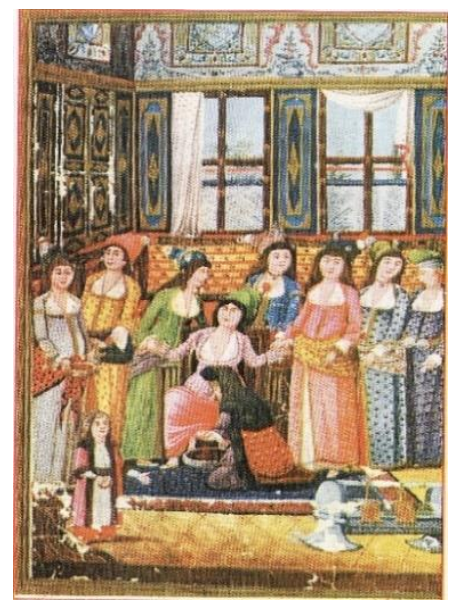

(10)

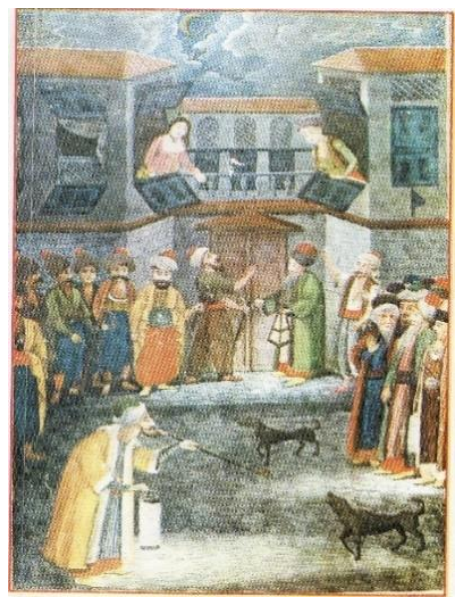

(شكل)

ان ما اغنى الحركة الفنية التشكيلية في كردستان العراق هو التوسع والانفتاح لمئات الفنانين الأكاديميين

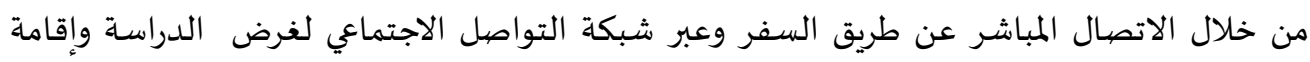

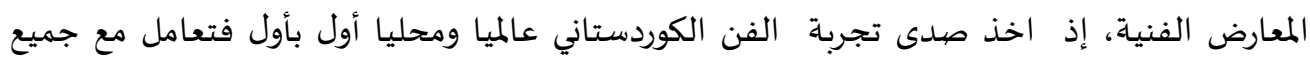

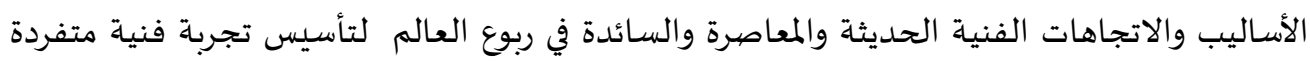

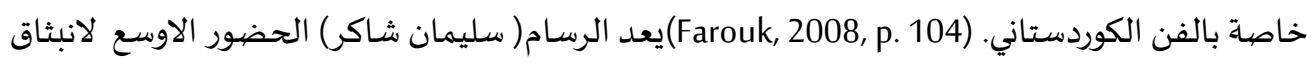

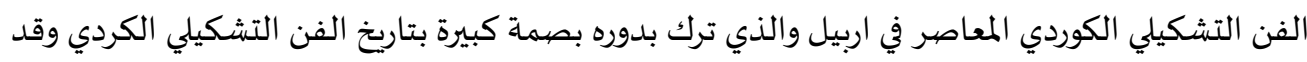
استطاع خلال نصف قرن من عمره أن يدرس كثير من الطلبة والفنانين من خلال تدريسه المراحل

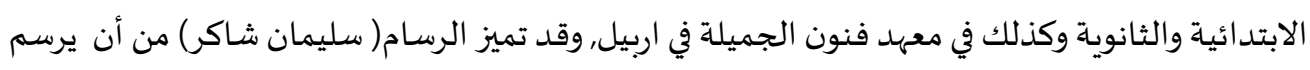
المناظر الطبيعة مع رسم الأشخاص بجمال وتنسيق وربط وتجسيد ألوانه ومكونات لوحاته الأخرى

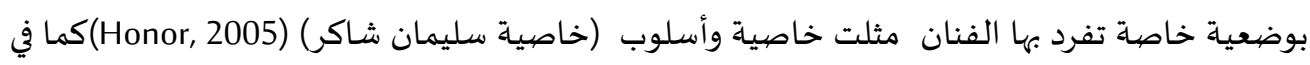




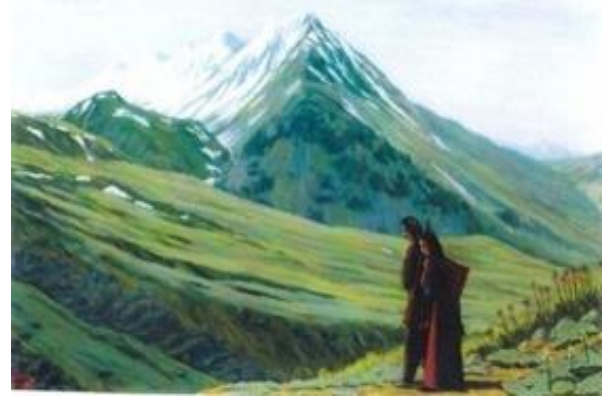

(شكل12)

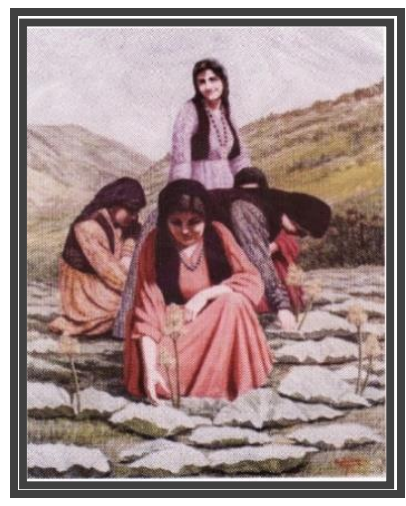

الشكل (11)

وكانت الأحداث التي مر بها المجتمع الكوردي قد شدته أكثر للوطن ومنحته شعوراً وحماسا قوياً بالأرتباط معاه وقد جسـدها بلوحات فنية عدة, تكتشف الخيط الذي يربطه مع الناس وإيمانه لضرورة الالتزام التهام الفني الواقعي ماقد

\section{2- الواقعية الاشتر اكية وتصوير الأبعاد الاجتماعية:}

لم يكن تصوير الفنان للواقع- مثلما سنجده عند( محمد عارف)- تصوير حرفيا مطابقا أي لم يكن ( نقلا

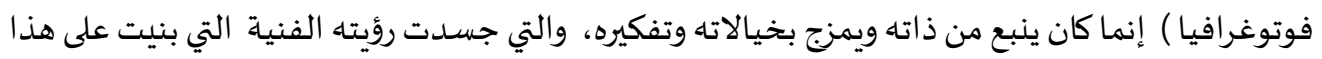

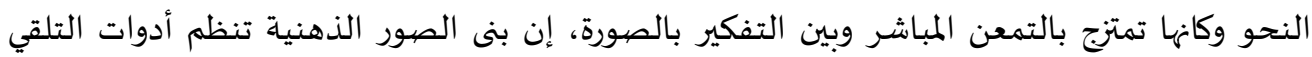

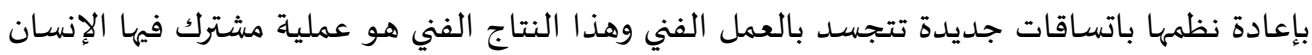

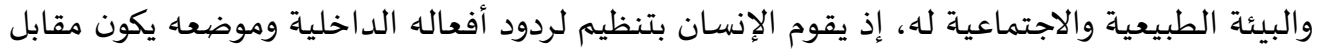

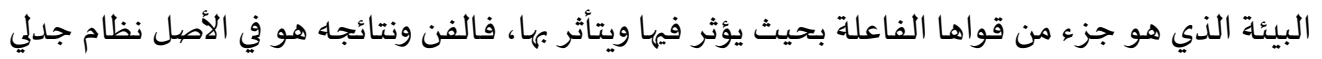

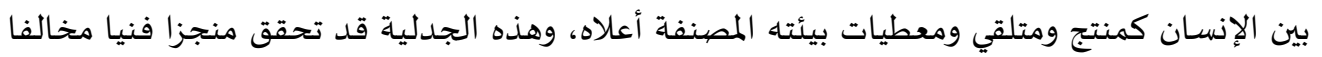

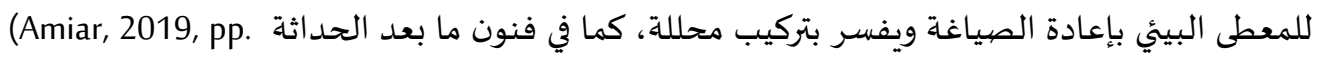

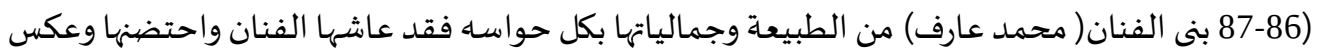

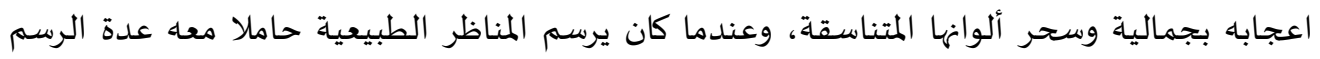

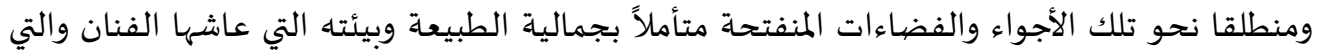
جسدها من خلال رسم ألوانها وأشعة الشمس وظلالها وأبعادها الساحرة متنقلا من موقع إلى آخر وهو

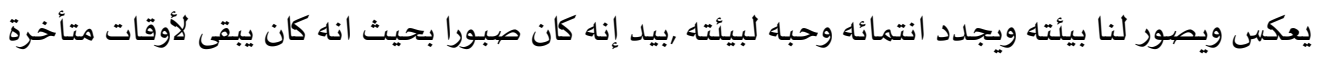

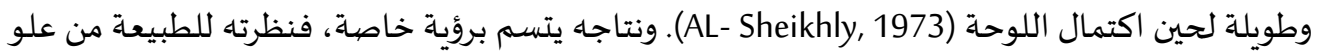
شاهق، لأنه يجلس مفتخرا على قمة جبل فيرسم الحياة بناسها وطبيعتها بجبالها ووديانها، و يمزجها

بخيالاته حين يتمعن بتلك المناظر الخلابة(Muhammad Aref,Nature Artist) كما في الاشكال (13-15) بهنيان 


\section{ISSN(Online) 2523-2029, ISSN(Print) 1819-5229 2021 مجلة الأكاديي-العدد 102-السنة}
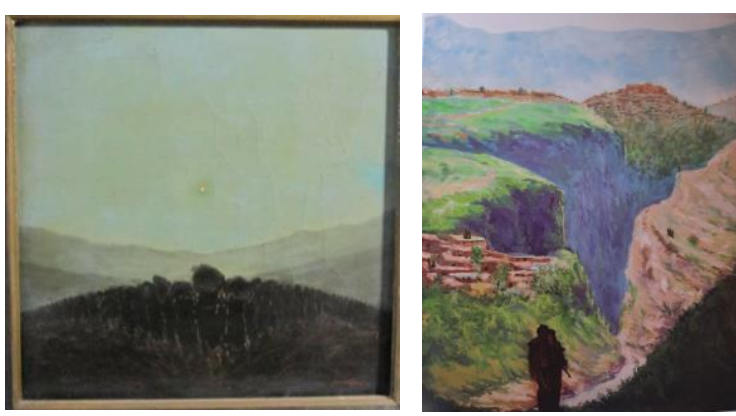

الشكل (15)

الشكل(14)

الشكل (13)

ولعل الأحداث التي تمر بها امتنا قد شدته أكثر للوطن ومنحته شعوراً قوياً بارتباطه معه وقد عبر عن ذلك

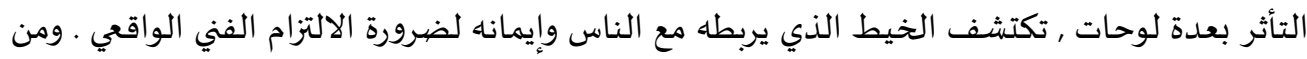

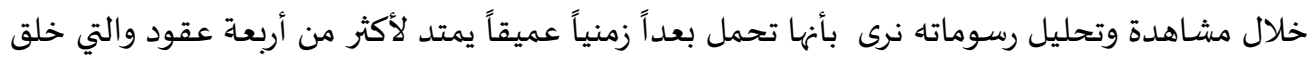
منها نقطة انطلاق مهماه شكلت هويته الفنية وتركت بصمتها الكبيرة في مسيرة المشهد التشكيلي العراقي المعاصر بشكل عام والفن الكوردي المعاصر بشكل خاص وذلك لما عكسته تجربته الفنية من شفافية وحساسية مرهفة عكست حرية الفنان استطاعت الجمع بين المعاني والعناصر الفنية اذ كان الفنان محمد

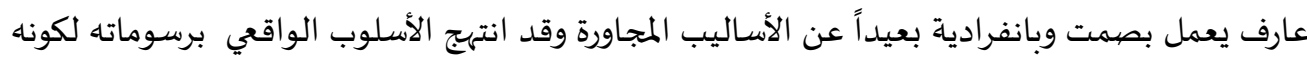

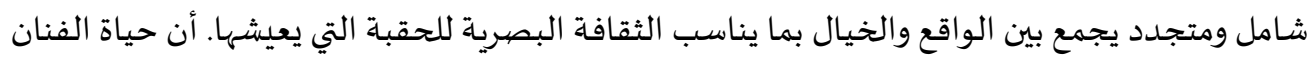

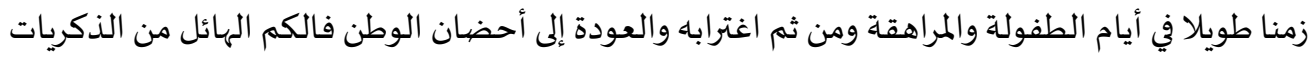
التي ظلت في داخله جسدها في أعماله بروح سامية وشعور داخلي متوهج وصديدا فيا بالتعبير أعمال الفنان التي مثلت الحنين إلى الوطن وتمكن أن يجسـد التعايش الكوردي العربي والقضايا الوطنية الأخرى وتعد التهد

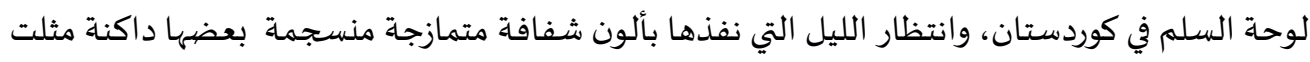
احساسه وعاطفته الذاتية وكذلك وحدة موضوعية مترابطة واهتمامه بالخطوط في إبراز الشكل تمثل

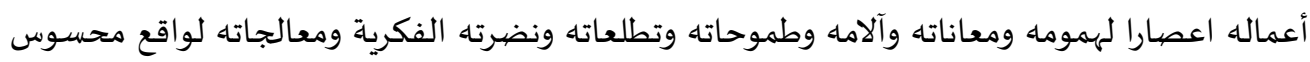

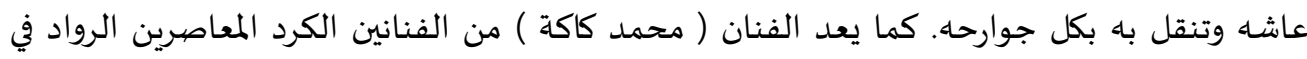

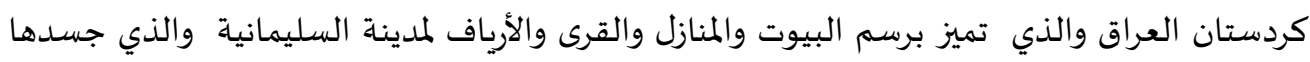

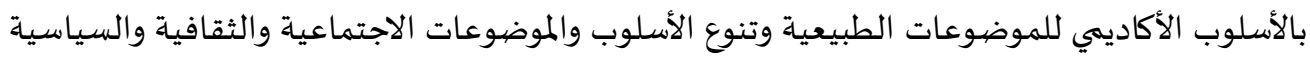

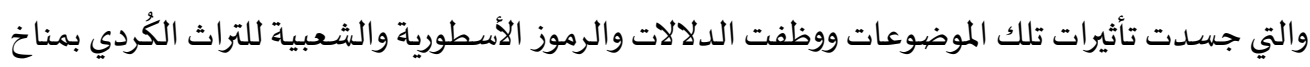
تاريني)(The Kurdish Plastic artist Muhammad Aref, 2012) كما في الاشكال (17-16) 
صاحب جاسم حسن البياتي - وائل نجم عبد. الـواقعية في رسومات فناني كردستان العراق.

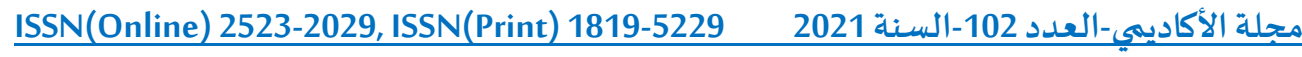

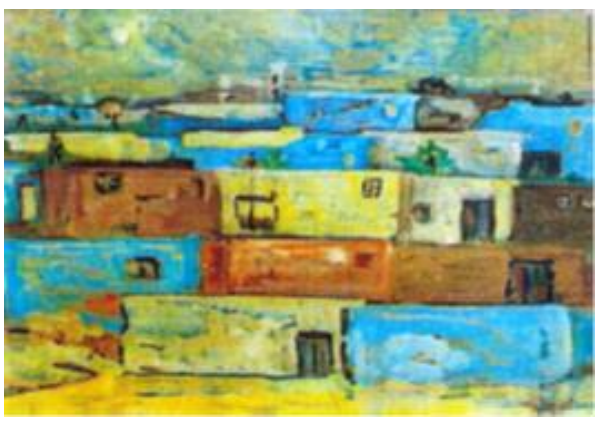

الشكل (17)

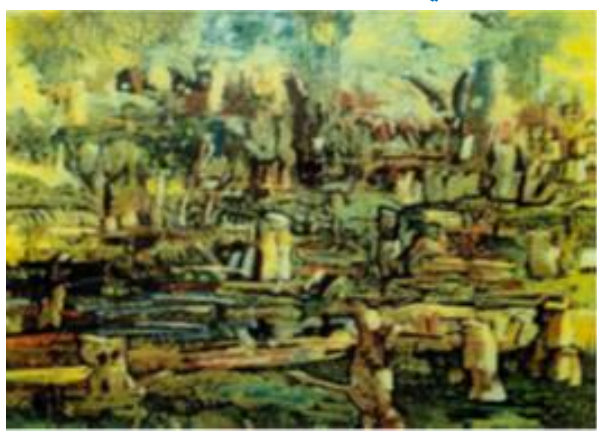

الشكل(16)

3-الـو اقعية النقدية وتناقض الموضهوعات السائدة:

في كتابه (الواقعية النقدية) وصفها (س بيتروف) بأهها أحد أصناف معرفة الحياة والتأثير عليها ، فالحياة

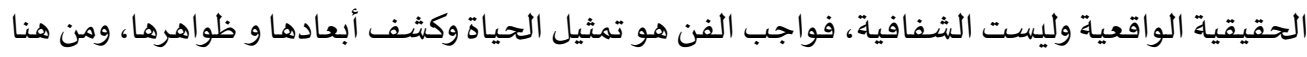

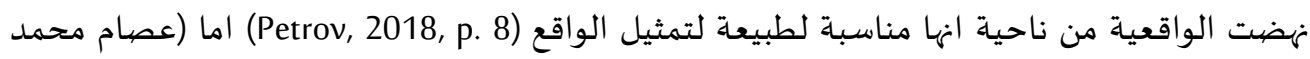

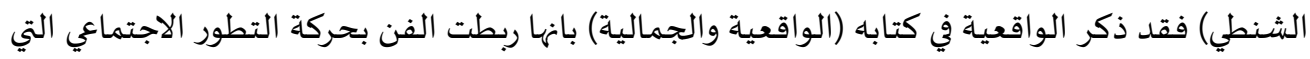

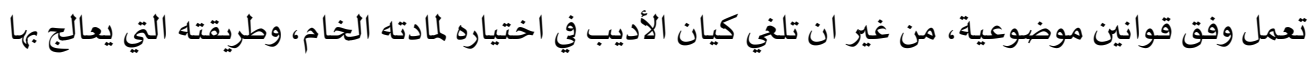

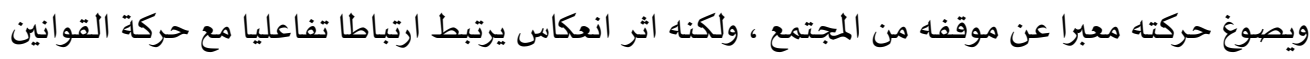

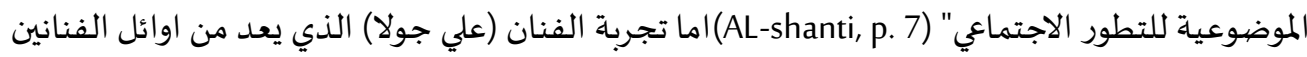

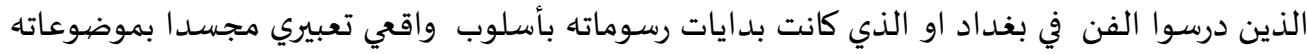

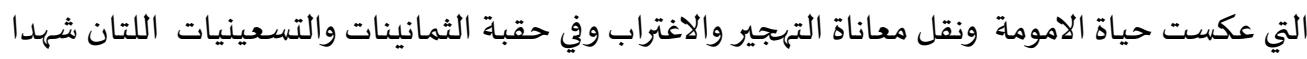

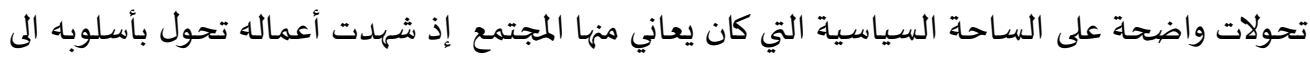

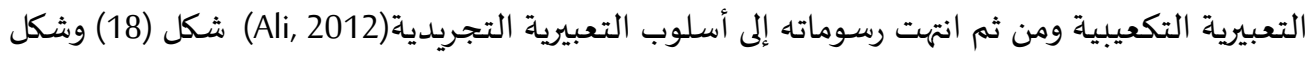

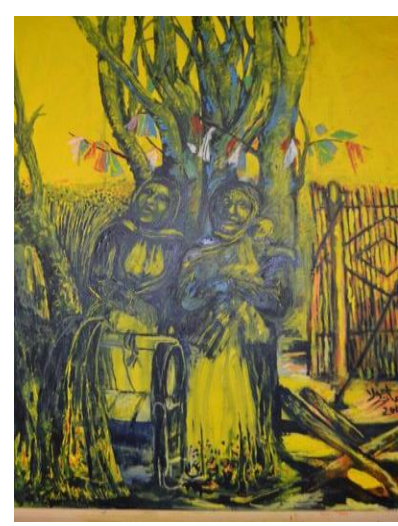

الشكل (19)

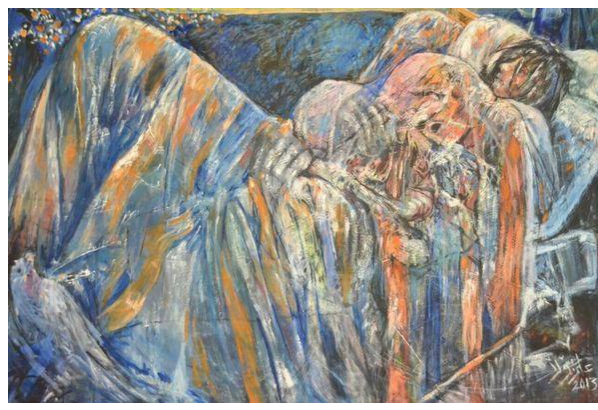

الشكل (18) (18) (2) (1) 
صاحب جاسم حسن البياتي - وائل نجم عبد الـواقعية في رسومات فناني كردستان العراق..إن. ISSN(Online) 2523-2029, ISSN(Print) 1819-5229 2021 مجلة الأكاديمي-العدد 102-السينة تميزت أغلب أعمال تلك الحقبة بالأسلوب الأكاديمي للموضيوعات الطبيعية وتنوع الأسلوب والموضوعات

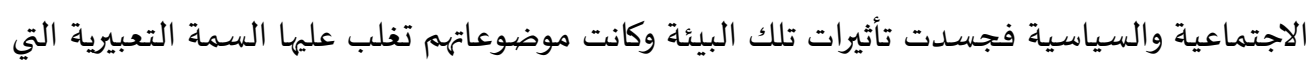

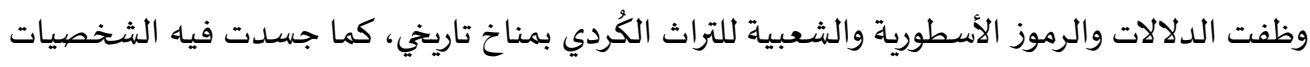
والقادة المؤثرين والمعروفين والحروب والثورات التي خاضوها وعدد من المنجزات الفنية التي كانت تحاكي

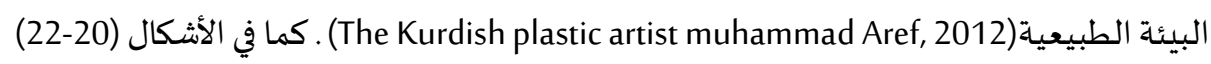

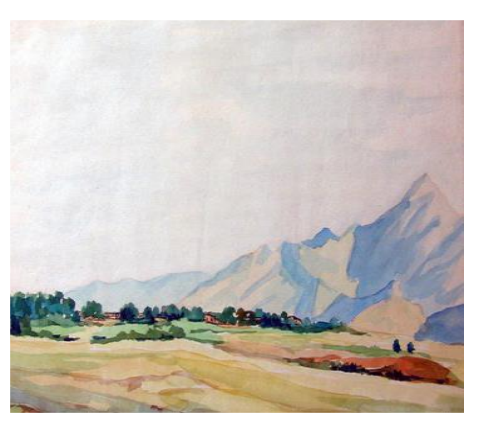

الشكل (22)

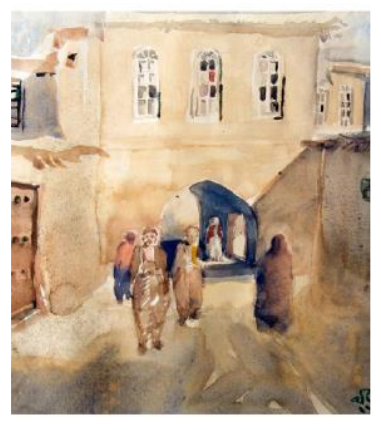

الشكل(21)

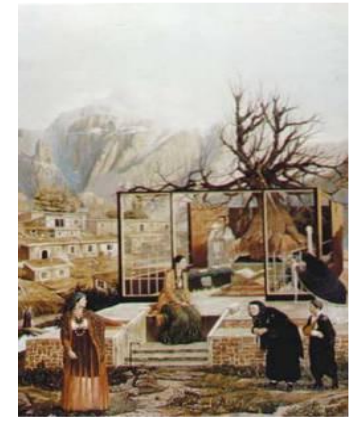

الشكل (20)

4- الـواقعية السحرية وإعادة صياغة المألوف: بحدود عمل الواقعية السحرية سلك( محمد عارف)

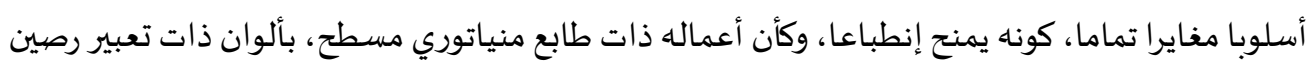

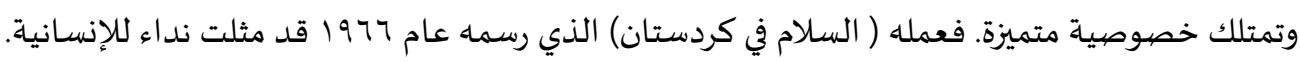

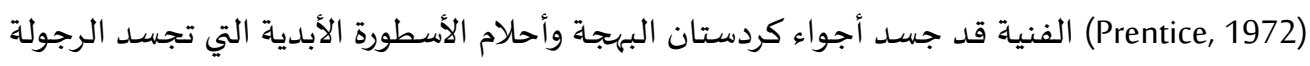
والشجاعة في ذات شخص بسيط يتلى الفنان باللغة التشكيلية قصة الإنسان والحب والوطن.. الخ كما في (23) الشكل)

لقد صور الفنان( عارف) في عدد من رسوماته صور لشخصيات (بورتريه) ولاسيما في حقبة اقامته خارج

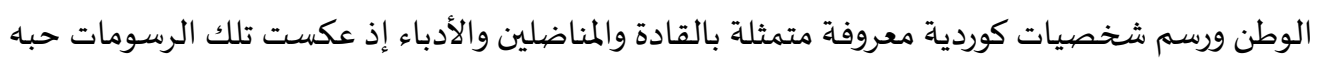

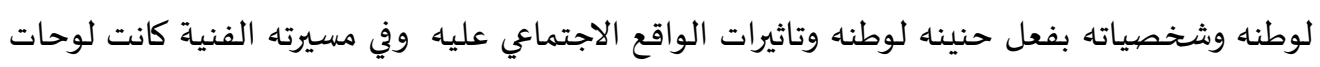

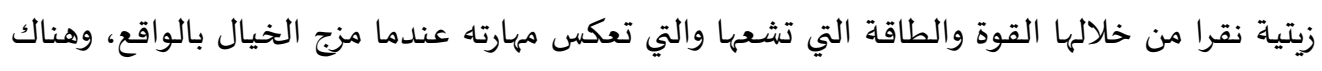
تواصل وترابط وثيق بين الفنان( محمد عارف) والجمهور وهناك تعاطف فيما بينهما، ببساطته التعبيرية

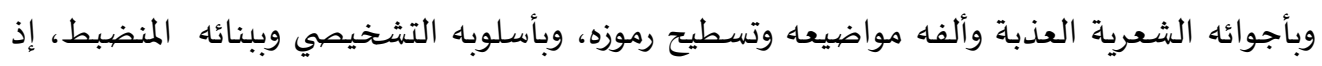

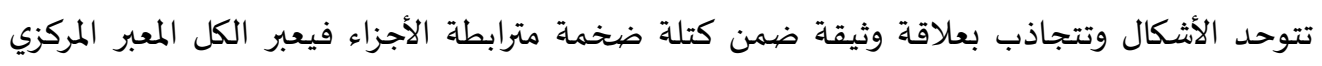
والرئيس عن خصوصيتها. فلا رغبة له بأن يغامر مع المغامرين الشكليين، أو مجازفات الفنانيين الأوربيين

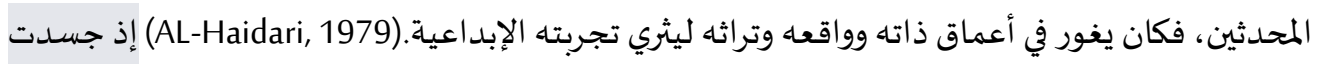

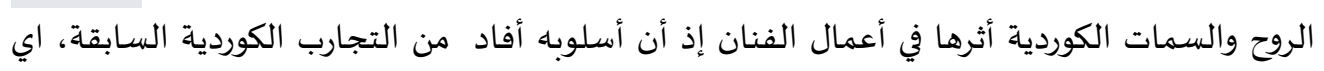

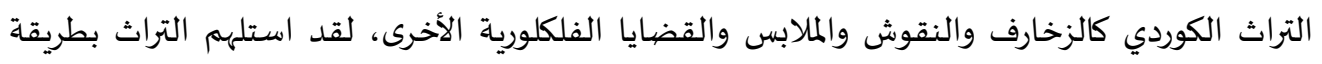


صاحب جاسم حسن البياتي - وائل نجم عبد الـواقعية في رسومات فناني كردستان العراق.

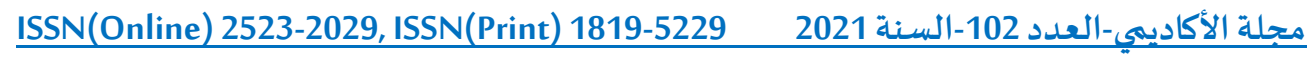
فكرية فعالة وهذه الطريقة لم تهيمن على أجواء العمل وحسب بل سخره بربط الماضي والحاضر بالمستقبل

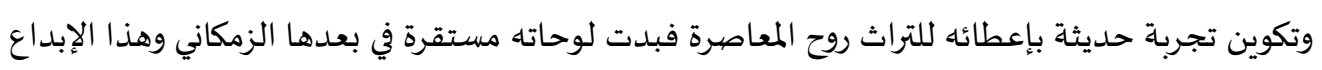

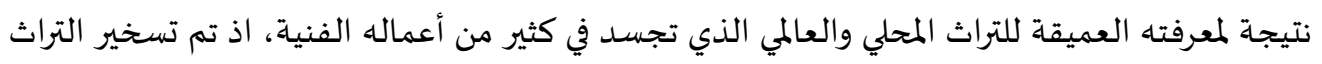

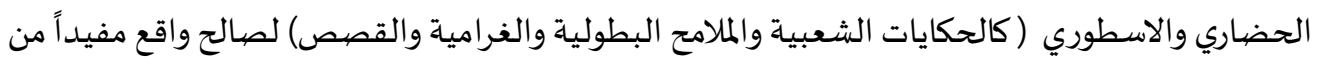

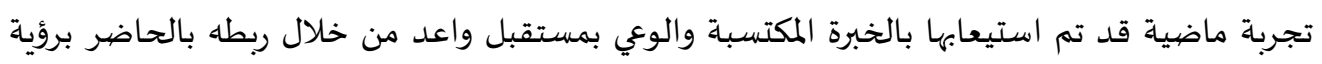

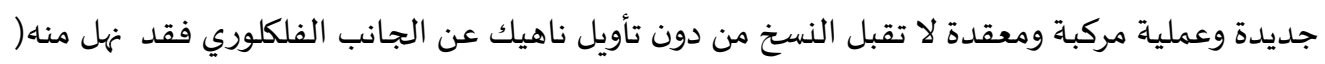

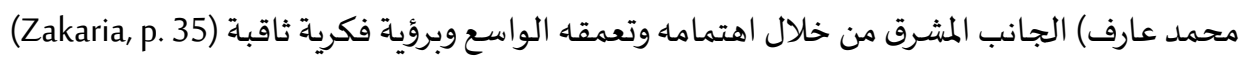
كذلك ظهرت تأثيرات الأساطير الكُوردية وانعكست في أغلب أعمال الفنان (ريبوار سعيد) كما في الشكل

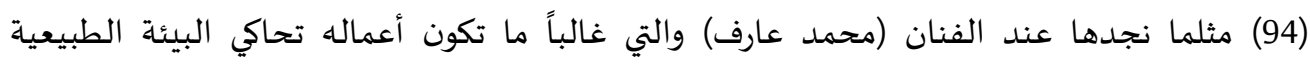

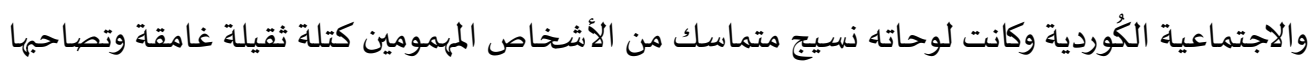

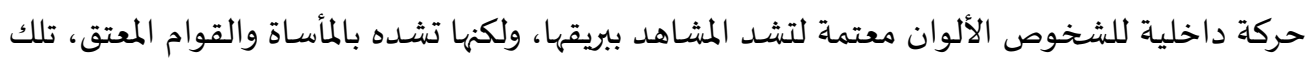

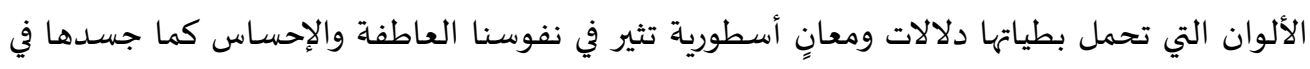

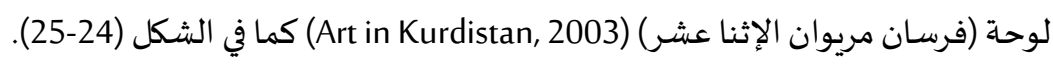

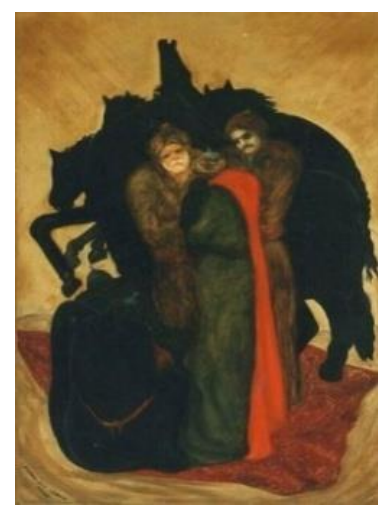

الشكل (25)

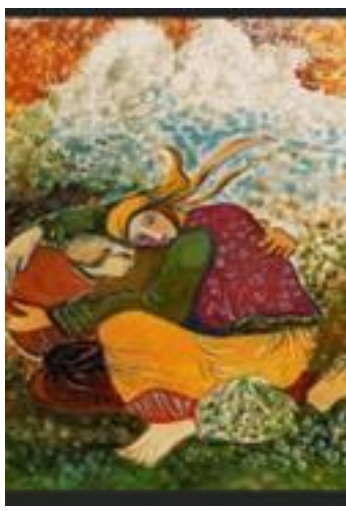

الشكل (24)

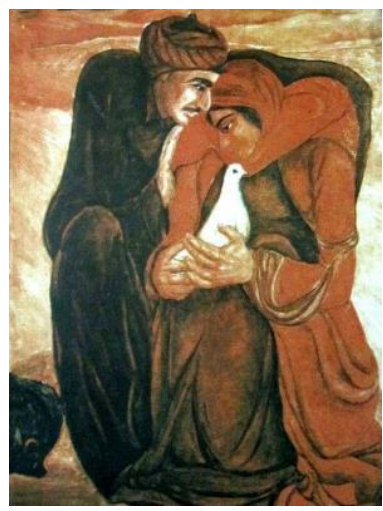

الشكل (23)

اما الفنان (جمال مشير) أحد الفنانين الكورد الواقعيين الذي كانت محاولات جادة ميزته في الكشف عن

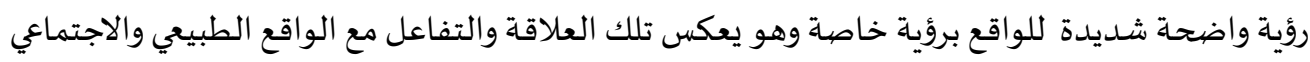

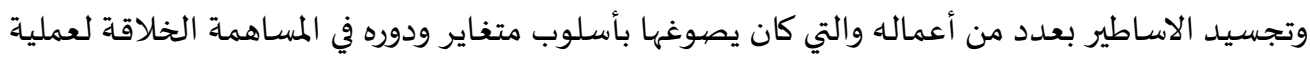

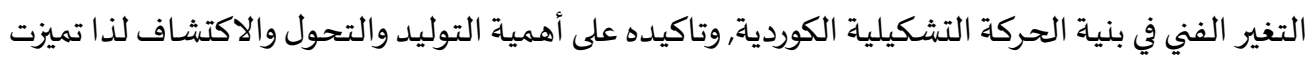

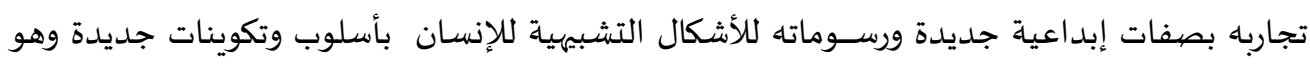

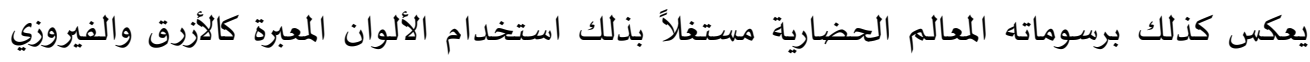
والبنفسجي والأحمر والأخضر كما في الاشكال (26-27) 


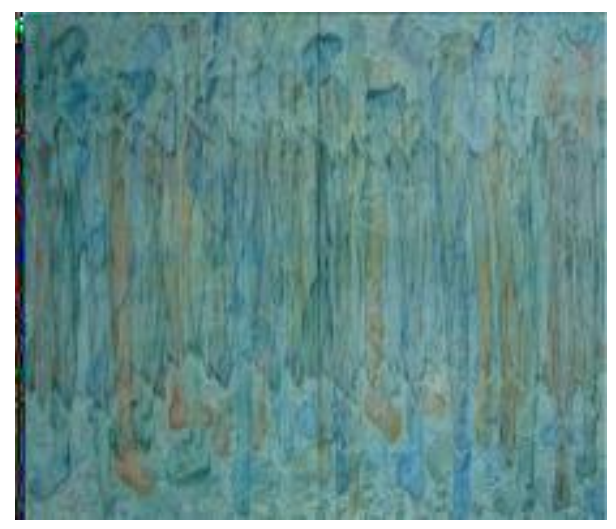

شكل(27)

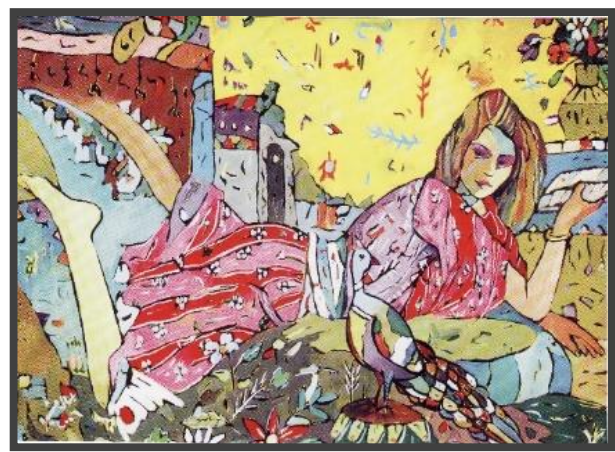

الشكل (26)

الفصل الرابع: نتائج البحث

1-وثقت الواقعية حياة المجتمع الكردستاني بما ينسجم مع تطورات العصر، إذ شهد الأسلوب سمة

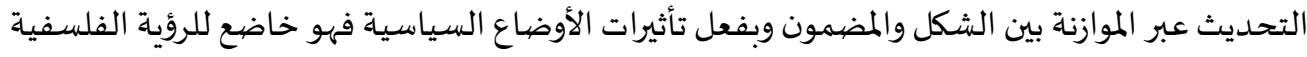
والأفكار الجدلية والمفاهيم.

2-تجسدت النزعة الرمزية والسحرية في الواقعية عبر فهم الواقع المحلي والتمتع بقيم الجمال واستلهام الرؤى البصرية الحداثوية.

3-اتجهت الواقعية ولاسيما في موضوعاتها التاريخية والأسطورية والبطولية بالأتجاه النقدي بفعل دينامية

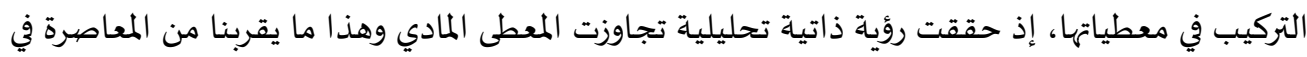
الفن.

4-حققت الواقعية-في بعدها النقدي- اكتشاف للبصيرة الفنية والفنتازيا الحالمة إزاء الأعمال الفنية التي تناولت الرمز والبطولة والأسطورة.

5-تميزت الواقعية بالبعد الأشتراكي في الموضوعات والمعالجات الفنية التي عكست البيئة الثقافية للمجتمع، فضلا عن الموضوعات التأريخية متضمنة حضيور المعاني والأفكار.

6-اعتمدت الواقعية على العالم الموضوعي الطبيعي على حساب الذات، فكان التصيوير منصباً على نقله

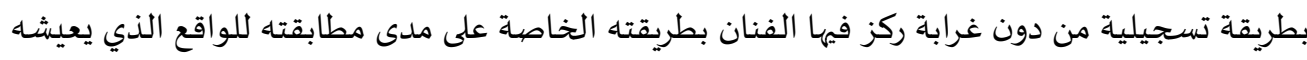
الإنسان.

7-تميزت الواقعية بالموضوعية بعيدة عن افكار الفنان ورؤاه الذاتية ولاسيما في اتجاهها الطبيعي فكانت أغلب الرسومات قد تناولت موضوعات المنظر الطبيعي والحياة اليومية للانسان. 


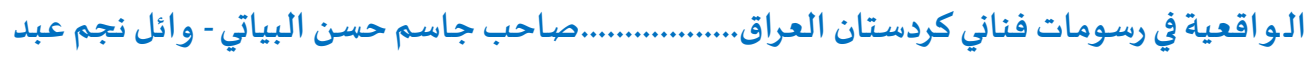
ISSN(Online) 2523-2029, ISSN(Print) 1819-5229 2021 مجلة الأكاديمي-العدد 102-السينة

8-تنوع أسلوب الواقعية الأشتراكية وبمختلف الموضوعات الأجتماعية والثقافية والسياسية والتي وظفت الدلالات والرموز الأسطورية والشعبية للتراث الكردي.

9-عكست الواقعية في رسومات كردستان موضوعات المعاناة والتهجير القسري فضلاً عن نتائج الحروب تحولاً أسلوبياً من الواقعية الطبيعية الى الواقعية النقدية. الأسـتنتاجات:

1-كان للظروف الأجتماعية والأقتصادية والسياسية في كردستان العراق دورا في بلورة المحتوى الواقعي للفن، الذي عد مرآة الحياة.

2-الحداثة في الفن هو تطوير للواقع الفني عبر استبدال النزعات الخيالية والأسطورية باتجاه صدق توثيق

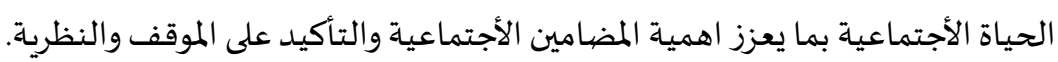

3- ان تعدد وتنوع أساليب التعبير في الواقعية يعد تفعيلاً لذاتية الفنان وفرديته في التعامل مع المتغيرات والأحداث السياسية والفكرية والأجتماعية.

4-لقد ساهمت كثير من الأحداث السياسية فضلا عن الأكتشافات العلمية الجديدة في البحث عن رؤى

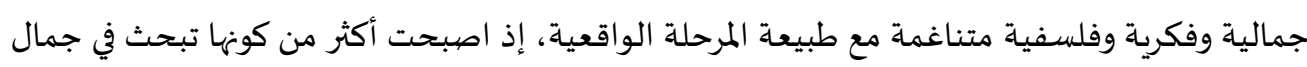

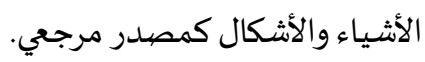

Referenes:

November, 1998 .(Raman Magazine,/ssue 29.)

Abd AL-Raqib Yusuf.(2005). The southern borders of kurdistan historically and geographically.Sulaymaniyah.

Adel Kamel .(1988) .Contemporary plastic Art in Iraq .Baghdad: House of General Cultural Affairs.

Adel Kamel.(2000) .Iraqi Formation, Foundation and Diversity .Baghdad: General cultural Affairs House.

Ahmed Fattah Sam.(2012). Aesthetic and Expressive values of portraits in Iraq Kurdistan Artists Drawings, Masters Thesis, college of Fine Arts university of sulaymaniyah . sulaymaniyah.

AL Said, S. H. (1983). Seasons of plastic movement in Iraq. Baghdad: Dar Al-Culture.

Amhaz, M. (1996). Contemporary artistic currents. Brut, Lebanon: Publications Company for Distribution and Publishing. 


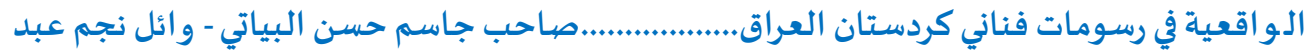

ISSN(Online) 2523-2029, ISSN(Print) 1819-5229 2021 مجلة الأكاديي-العدد 102-السينة

Annan, L. (n.d.). The realism in French Literature . Cairo: Dar Al- Maaref.

Art in Kurdistan.(2003) .altaakhipress.com.

Atyaf ali najm lyad Mahmoud Haidar.(2017) .Features cosmicism in contemporary Iraq formation .Al- Academy Journal Issue 82, college of Fine Arts, university of Baghdad. p52.

Bland AL-Haidari) .March, 1979 .(From The artists notebook on the occasion of the seventh solo exhibition .National Museum Hall.

Boris, B. (n.d.). Realism today and Foraver.

Fadl, S. (1980). The Realism Approach in Literary Creativity. Cairo: Dar Al Maaref For Printing and publishing.

Gatchev, G. (1990). Consciousness and Art. Kuwait: The world of Knowledge.

George, B. (1977). Art and physical History. Beirut: Dar AL-talia.

George, L. (1970). Studies in Realism. Damascus: publication of the Ministry of Culture.

Hassan, H. M. (n.d.). Contemporary Art doctrines. Cairo: Dar Al-Fakr Al- Arab.

Hassan, Sahib jassim.(2017). The constructivist transformer from the installation systems in the work of the artist Ali AL-Najjar . Al-Academy Journal, Issue 83, College of Fine Arts, university of Baghdad.p41.

Hussein AL-Husseini.(1984) .Ismail AL-sheikhly .Arab Horizons Magazine. p35.

Ibrahim Jabra Jabra.(1972) . Contemporary Iraqi Art Baghdad.

Ibrahim jabra Jabra.(1986). The Roots of iraqi Baghdad: Al- Arabiya House.

Ibrahim Zakaria ..Art Problem .EGypt: Dar Misr for printing.

Ismail AL-Sheikhly.(1973). AL-Thawrw newspaper.the number 1415.

Issam Mahmoud AL-shanti ..Aesthetics and Realism in our modern criticism .Beirut,Lebanon:

The Arab institute for studies and publishing.

Jamal Rashid .(1973) in the beginning was Hassan Falah .AL-taakhi newspaper.

Jamal Rashid, Fawzi Rashid Ahmed.(1990) .the ancient history of the Kurds .Erbil :Ministry of Higher Education and scientific Research.

Kamal Hussein Amiar .(2019) .Plastic Artist pioneer profssor Muhammed Aref.Erbil: Tehran press,Edittion 1.

Karim Ali .April, 2012 . (Journal of Al-sulaymaniyah Exhibitionsm,(Haocarrie) namber(171.(

Mahdi, A. N. (2012). pioneering European painting styles. Baghdad: . Al- Academy Journal College of Fine Arts, University of Baghdad. 


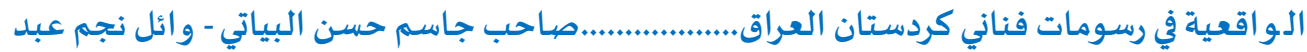

ISSN(Online) 2523-2029, ISSN(Print) 1819-5229 2021 مجلة الأكاديي-العدد 102-السينة

Muhammad Aref,Nature Artist .almadasupplements.com/print.php?cat=8031.

Natiq, K. (2008). Reading in the term. Baghdad: General Cultural Affairs House.

Nizar Salim ..Ismail AL-Sheikhly, The moernity of painting and the aesthetics of discourse.

Ofsianikof. (1987). Aesthetic Values. Alexandria University: University Knowledge House .

Peter, B. (2019). Modernity And Postmodernism. The Cultural Foundation Publications.

Petrov.(2018) .Monetary realism .Syrian: Syrian General Book Authority.

Prentice.(1972) . German Democratic Art Magazine.

Qasim Mohsen Hassan.(2016) .Metaphor in Eaeq Hassan Drawings. Al- Academy Journal

Issue 80, college of fine Arts, university of Baghdad.p24.

Renee, H. (1978). Art Hs interation and its Way, part Two. Syria, Damascus: Ministry of Culture Publications.

Roger, G. (1968). Realism Without Banks. Cairo: Arab Writer House.

Saber Muhammad Farouk.(2008). Anfal campaigns in the drawings of Iraqi Kurdistan artists .

Sulaymaniyah: universty of sulaymaniyah, college of fine Arts.

Saleh, I. N. (2014). Intellectual Systematicism in Realistic plastic Art Works, Paintings by

Iranian Artist Ayman Al-Maliki. . Al- Academy Journal, College of Fine Arts, University of Baghdad, p. 46.

Shaker Hassan AL Said.(1980) .The origin ad Devlopment of fine art in Iraq .Arab Horizons

Magazine.p120.

Shaker Hassan AL-Said .(1994) . Articles in theorization and Art criticism . Baghdad: House of

General Cultural Affairs.

Shawkat AL-Rubaie.(1976) .Painting and ideas .Baghdad: AL- Hurriya Publishing House.

Shawkat AL-Rubaie.(2002) .Isail AL-Sheikhly, Modernity of painting and Aesthetics of

Discourse .Baghdad: Association of Iraqi Plastic Artists.

Sydney, F. (1971). Realism in Art. Cultural press.

Tain, H. (1963). Philosophie de art. Mexico: traduction, Espanol.

Tara Honor.(2005) .Modernity and Art .Erbil: Aras press for publishing and Distribution.

The Kurdish plastic artist muhammad Aref.(2012). AL-Taakhi newspaper,Tuesday.

The Kurdish Plastic artist Muhammad Aref.(2012). AL-takhi newspaper.

Wellck, r. (1968). Conceptos de Criticn Litearia. Venzuela: al Espanol. 


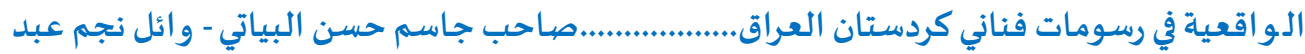

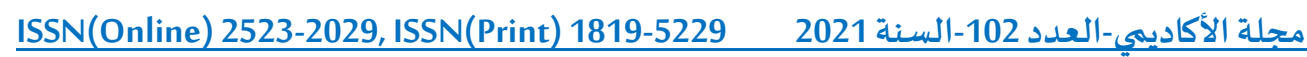

DOI: $\underline{\text { https://doi.org/10.35560/jcofarts102/177-194 }}$

\title{
Realism in the painting of Iraqi Kurdistan artists "A Study in methods of expression"
}

\author{
Sahib Jasim Hassan ${ }^{1}$ \\ wael Najm Abd ${ }^{2}$
}

Al-Academy Journal

Issue 102 - year 2021

Date of receipt: $18 / 10 / 2021$.

...Date of acceptance: $29 / 11 / 2021$......Date of publication: $15 / 12 / 2021$

\begin{abstract}
:
The tagged research (realism in the Paintings of Iraqi Kurdistan artists, "a study of expression methods") dealt with realism in an objective way, as well as the complexity of its concepts through its formations and formations. On realism and its historical dimension in concept and meaning, as for the second chapter, the research was focused on the methods of expression in painting, while the third chapter was concerned with the procedural applications of realistic methods of expression in the drawings of Iraqi Kurdistan, and according to these axes and to achieve the goal of the research, a number of Among the results are:

1- Realism documented the life of the Kurdish society in line with the developments of the era, as the style witnessed the feature of modernization through balancing between form and content and due to the effects of the political situation, as it was subject to philosophical vision, dialectical ideas and concepts.

2- Realism achieved in its critical dimension a discovery of artistic insight and dreamy fantasy about artworks that dealt with symbol, heroism and legend.
\end{abstract}

key words:

Realism, styles, expression, Iraqi Kurdistan, Kurdistan artists.

\footnotetext{
${ }^{1}$ University of Baghdad / College of Fine Arts, sahib_hassin@yahoo.com .

${ }^{2}$ Ministry of Education, wailnajem07@gmail.com .
} 


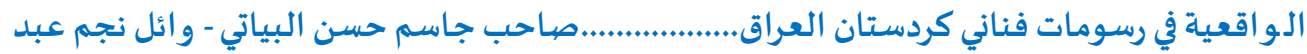

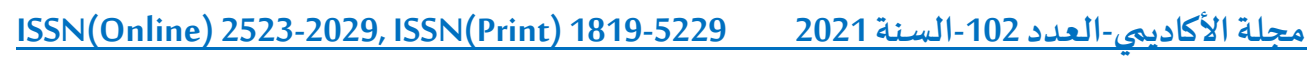

\section{Conclusions:}

1- The social, economic and political conditions in Iraqi Kurdistan played a role in crystallizing the realistic content of art, which was considered the mirror of life.

2- Modernity in art is the development of artistic reality by replacing imaginative and mythical tendencies towards the authenticity of documenting social life in a way that enhances the importance of social contents and emphasizes the position and theory.

3- The multiplicity and diversity of methods of expression in realism is an activation of the artist's subjectivity and individuality in dealing with political, intellectual and social changes and events.

4- Many political events, as well as new scientific discoveries, have contributed to the search for aesthetic, intellectual and philosophical visions in harmony with the nature of the realistic stage, as it has become more than looking at the beauty of things and forms as a reference source. 